Volume: 2 | Volumen 2 | Número 2 | Number 2 | pp 191 - 207 ISSN: 2634-355X (Print) | ISSN: 2634-3568 (Online) journals.tplondon.com/yeiya

First Submitted: 29 November 2021 Accepted: 30 November 2021 TRANSNATIONAL PRESS ${ }^{6}$ DOI: https://doi.org/10.33182/y.v2i2.2017

\title{
E1 trabajo como elemento constitutivo de la masculinidad: abordajes en el contexto laboral fronterizo-migratorio entre los mixtecos en Tijuana
}

\author{
Christian Arnulfo Angeles Salinas ${ }^{1}$
}

\section{Resumen}

Desde el enfoque de los estudios de género de los hombres, las masculinidades y la masculinidad, este artículo pretende analizar las maneras en que los hombres mixtecos oriundos de San Miguel El Grande, Tlaxiaco, Oaxaca, residentes en Tijuana, construyen y significan su masculinidad a partir del trabajo, enmarcados por procesos socioculturales basados en las relaciones de género. Se pone particular énfasis en los sistemas de relaciones sociales, el simbolismo y el poder que reflejan los ordenamientos de género en los grupos sociales, mismos que se redefinen no sólo en las experiencias de los hombres y las mujeres sino también en el marco de procesos sociales, de cambios generacionales y de residencia de contextos comunitarios/rurales a contextos urbanos/fronterizos.

Palabras clave: Masculinidad; Trabajo; Relaciones de Género; Migrantes; Mixtecos

\section{Work as constituent element of masculinity, approach from the border-migrant laboral context among mixtecs in Tijuana}

\begin{abstract}
This paper aims to analyze the ways in which Mixtec men from San Miguel El Grande, Tlaxiaco, Oaxaca, who are residents of Tijuana, build and signify their masculinity by means of the the labor/work. framed by sociocultural processes based on gender relations. Special emphasis was placed on the systems of social relations, power and symbolism visible through the gender order in social groups. The topic was addressed based on the Gender studies of men, masculinities and masculinity
\end{abstract}

Keywords: Masculinity; Labor/Work; Gender relations; Migrants; Mixtecs

\section{Introducción}

El presente documento es el resultado de un proceso reflexivo sobre la investigación realizada durante 2014-2018 en la cual se buscó conocer cómo un grupo de varones pertenecientes a un grupo indígena con amplia tradición migratoria construía y significaba sus masculinidades a partir de elementos como el ejercicio de poder a través de las mayordomías, la paternidad, el trabajo y la sexualidad. Se ha decidido ampliar el análisis respecto al trabajo como uno de los mandatos sine qua non de la masculinidad, para dar cuenta de las transformaciones,

\footnotetext{
${ }^{1}$ Doctor en Ciencias Sociales con especialización en Estudios Regionales por El Colegio de la Frontera Norte (EL COLEF), México. Actualmente es Investigador Posdoctoral en el Centro de Investigación en Ciencias Sociales y Estudios Regionales de la Universidad Autónoma del Estado de Morelos (UAEM), México. Correo: angeles.christian@gmail.com.
} 


\section{El trabajo como elemento constitutivo de la masculinidad}

continuidades y ajustes que, desde la masculinidad, realizan algunos varones para mantener el dominio masculino entre este grupo de estudio en la ciudad fronteriza de Tijuana.

El trabajo ofrece a los hombres cumplir con el mandato masculino de la proveeduría (Valdes, T. \& Olavarria, 1997), pero no sólo eso, el trabajo es una manera de mostrar el conocimiento sobre el trabajo y habilidades para el uso de las herramientas. Es decir, permite mostrar el desempeño laboral de los varones el cual está en función de la eficiencia y los resultados de este (Gilmore, 1997). Este artículo busca conocer cómo algunos varones mixtecos, oriundos de San Miguel El Grande, Tlaxiaco, Oaxaca (SMG) que residen en Tijuana, construyen y significan su masculinidad a partir del trabajo que realizan, a través del cual interactúan en términos de la organización social y comunitaria, entre generaciones (jóvenes, adultos y ancianos) y experirncias de migración (nacional e internacional).

El artículo se divide en cinco partes, en la primera se describe el marco analítico el cual está basado en la perspectiva de género que permite desglosar las categorías de masculinidad y trabajo y su traslape para la construcción y significación de la masculinidad en tanto identidad de género. En la segunda parte se elabora una descripción de los procesos socioculturales que enmarcan la construcción y significación de la masculinidad a partir del trabajo y del desempeño laboral. Posteriormente se hace una exposición de la cuestión metodológica y del acceso a la información con la comunidad mixteca en Tijuana. En la cuarta sección se muestra y analiza la evidencia etnográfica que permite ahondar en la construcción y significación de la masculinidad entre este grupo de varones de SMG. Finalmente se exponen las conclusiones y algunas vetas descubiertas para dar continuidad a este tipo de estudios.

\section{Marco analítico}

En este artículo se retoma como marco analítico los estudios de género de los hombres, la masculinidad y las masculinidades, los cuales, de acuerdo con (Núñez, 2016, pp. 20-21):

Recuperan la perspectiva de género planteada por las feministas y parten de la consideración de que los varones somos sujetos genéricos, esto es, que sus identidades, prácticas y relaciones como hombres son construcciones sociales y no hechos de la naturaleza, como los discursos dominantes han planteado por siglos [...] los estudios de género de los hombres y las masculinidades ubican a los hombres como sujetos dentro de un sistema sexo-género, un sistema de ideologías, identidades y relaciones androcéntricas y heterosexistas, que son nuestra actual herencia cultural.

En consecuencia, se retoma al género como categoría analítica (Scott, 1996), a través de la cual es posible analizar los sistemas de relaciones sociales en donde el ejercicio de poder entre los sexos se hace evidente. La aportación de Scott (1996, p. 281) respecto al género como categoría analítica llama a "prestar atención a los sistemas simbólicos, esto es, a las formas en que las sociedades representan el género, hacen uso de éste para enunciar las normas de las relaciones sociales o para construir el significado de la experiencia". Además, a partir del trabajo de Butler (2001), se considera la importancia de "cuestionar las cuestiones [sic] de poder que condicionan y limitan las posibilidades dialógicas” (Butler, 2001, p. 48), con la finalidad de comprender las diferentes acciones e interacciones de los sujetos de estudio en diferentes situaciones de la vida diaria en el contexto sociocultural en el que se construye y reproduce la masculinidad. 
Por lo tanto, se asume que, entre los hombres mixtecos abordados como sujetos de género, la construcción de la masculinidad "como fenómeno variable y contextual [...] no denota a un ser sustantivo, sino a un punto de convergencia relativo entre series de relaciones culturales e históricas específicas" (Butler, 2001, p. 43). Es decir, la masculinidad, su construcción y significación dependen del ejercicio de poder que se visibiliza en el entramado de relaciones e interacciones en las que los hombres participan y establecen relaciones de podersubordinación derivadas de una posición de clase, generación y/o pertenencia a grupos indígenas que ocupan en la sociedad.

En este escenario, estudiar a los varones de este grupo social implica un abordaje desde los estudios de género de los hombres, la masculinidad y las masculinidades, poniendo particular énfasis en los sistemas de relaciones sociales, el simbolismo y el poder que reflejan los ordenamientos de género en los grupos sociales, mismos que se redefinen no sólo en las experiencias de los hombres y las mujeres - pertenezcan a un grupo indígena o no- sino también en el marco de procesos sociales, de cambios generacionales y de residencia de contextos comunitarios/rurales a contextos urbanos/fronterizos palpables a través del discurso que tanto varones, como mujeres elaboran. Además de los simbolismos y acciones en las interacciones entre los sujetos en escenarios de la vida diaria, implica la problematización de las realidades generizadas (gendering) desde el patriarcado y el heterosexismo como elementos centrales del despliegue colonizante del sistema mundo occidental. $^{2}$

\section{Masculinidad}

La masculinidad ha sido conceptualizada desde diferentes enfoques, Valdés y Olavarría (1997, p. 11-12) resumieron las seis perspectivas propuestas por Clatterbaugh: la perspectiva conservadora, que considera natural que los hombres sean los proveedores y protectores de las mujeres; la perspectiva profeminista, que subraya que la masculinidad ha sido creada a través del privilegio de los hombres y la correspondiente opresión de las mujeres; la perspectiva de los Men's Rights, que postula que los roles masculinos tradicionales son altamente dañinos y que los hombres son víctimas de ello; el llamado movimiento mitopoético, que se basa en la convicción de que la masculinidad deriva de patrones inconscientes profundos, los que se revelan a través de las leyendas, mitos y rituales y que requieren ser actualizados por los varones; la perspectiva socialista, que señala que el capitalismo patriarcal define masculinidades asociadas a los tipos de trabajo y al control del trabajo de otros; $\mathrm{y}$, la perspectiva de grupos específicos, que enfatiza la existencia de una diversidad de experiencias que abarca no sólo a los hombres blancos, sino también a negros y grupos étnicos, heterosexuales y homosexuales.

La perspectiva con la que se aborda este trabajo busca tener el alcance de la conceptualización profeminista, pues los ejercicios de poder en las relaciones de género han redundado en

\footnotetext{
${ }^{2}$ El género es un proceso en constante transformación. West \& Zimmerman (1987) argumentaron que el género es un proceso, son acciones derivadas de ordenamientos sociales y por ende no son puntos de partida ni de llegada. Aludir al género como categoría analítica implica aludir a construcciones sociales imbricadas en los grupos sociales visibles en las relaciones de género, las cuales a su vez están mediadas por relaciones de poder.
} 


\section{El trabajo como elemento constitutivo de la masculinidad}

privilegios para los varones, ${ }^{3}$ y con ellos los varones significan su masculinidad. Además, al utilizar al género como categoría analítica en donde la cuestión relacional juega un papel relevante, es posible alcanzar un conocimiento con mayor profundidad del objeto de estudio, pues se abarca una mayor comprensión de la totalidad social al reconocer la diversidad de experiencias en torno a los varones y la masculinidad, tales como la construcción de ella desde los aspectos vinculados a procesos comunitarios o de la diversidad sexual.

\section{Trabajo}

Se retoma al trabajo como núcleo de construcción de la masculinidad. El trabajo forma parte fundamental de la construcción y significación de ésta, así lo han hecho notar diferentes autores (Valdés, T. \& Olavarria, 1997; Salguero, 2007; Hernández, 2010). Por ejemplo, Salguero (2007) en su estudio en México sobre varones profesionistas con licenciatura y maestría con nivel socioeconómico alto, afirma que el trabajo "forma parte de la identidad masculina; desde temprana edad, los niños crecen con la idea de que, a través del trabajo, serán reconocidos como hombres, dedicando gran parte de su vida a lograr un aparente éxito profesional y laboral" (Salguero, 2007, p. 430).

En cambio Olavarría (1999), en una revisión de diferentes trabajos sobre construcción de la masculinidad, encontró que es durante la etapa de adolescencia y juventud en donde los varones tienen que demostrar que son "verdaderos hombres". El autor identifica al trabajo como uno de los mandatos más determinantes de su vida, pues "trabajar significa ser responsable digno y capaz, atributos que caracterizarían a la hombría en su fase adulta plena" (Olavarría, 1999, p. 2). Finalmente, Hernández (2010), en un estudio sobre las transformaciones socioecónomicas estructurales y su impacto en las identidades masculinas y redefinición de la masculinidad entre los varones de Ciudad Victoria, Tamaulipas, afirmó que:

El trabajo, entonces, más allá de ser una práctica física, de subsistencia, es una práctica simbólica que moldea los significados de ser y actuar como un hombre, que se asocia con la capacidad de ser un proveedor económico y un jefe de familia que tiene la responsabilidad de mantenerse y mantener a otros, además de ser reconocido socialmente (Hernández, 2010, p. 248).

El trabajo es, entonces, un elemento constitutivo y fundamental de la masculinidad, le da sentido a la existencia vital y cotidiana de los hombres. Así mismo, el trabajo se socializa entre los varones desde la infancia, y de la mano de éste se construye la masculinidad a través de la proveeduría. Además, este proceso de construcción de la masculinidad a partir del trabajo es visible de manera constante entre los diferentes estratos sociales. Sin embargo, para los fines de este artículo, el trabajo se entiende como aquellas actividades remuneradas económicamente a través de las cuales los hombres muestran frente a la comunidad y a otros hombres y mujeres conocimiento, habilidades, arrojo y valentía. El trabajo entre los mixtecos va más allá de ser un elemento con el que los hombres se significan como proveedores de su

\footnotetext{
${ }^{3}$ A principios de los años 1970 aparecen grupos de hombres simpatizantes con el feminismo que sostienen que la masculinidad se crea y mantiene a través de una lógica de dominación que otorga privilegios para los hombres, y que conllevan a la opresión de las mujeres. Hay matices dentro de esta perspectiva: algunos enfatizan en los privilegios que el sistema patriarcal otorga a los hombres en el nivel macro, y otros profundizan en las limitaciones que los papeles de género tienen para la realización personal de hombres y mujeres (Faur, 2004, p. 43).
} 
familia y como hombres responsables como lo argumentan Gutmann (2000), Salguero (2007) y Hernández (2009).

Sin embargo, hay una faceta del trabajo que no ha sido abordada plenamente, la cual tiene que ver con la eficiencia y los resultados derivados del trabajo, es decir, el desempeño laboral. El desempeño laboral es un aspecto del trabajo que los hombres retoman para la construcción y significación de la masculinidad y éste va más allá de trabajar para proveer a una familia. Ttrabajar significa mostrarse ante la comunidad, otros hombres y mujeres a través de la actividad laboral desarrollada y visible a través del conocimiento o valentía demandado para realizarla y no por el resultado estrictamente económico.

El proceso de inserción de las mujeres al mercado laboral formal entre esta comunidad mixteca radicada en Tijuana trajo como consecuencia que el trabajo de los varones se viera rebasado como significativo respecto a la cuestión de la proveeduría, por lo que la construcción y significación de la masculinidad se verá reflejada en el campo del desempeño laboral. Para este artículo, el trabajo es un conjunto de actividades en las que se desarrollan prácticas, saberes y representaciones de masculinidad que no sólo dan la capacidad de proveer, sino que marcan la pauta para desarrollar competencias entre los varones para significarse a través de la dominación, la fortaleza, la fuerza corporal y la superioridad respecto a los otros hombres.

\section{El trabajo como elemento constitutivo de la masculinidad}

Se puede afirmar que existe una masculinidad dominante la cual tiene como principales expresiones en la heterosexualidad la paternidad y el trabajo (Valdés y Olavarría, 1997). Ser padre y ser proveedor son dimensiones constantes en el esquema dominante de las identidades de género masculinas. Lo primero atiende a aspectos de heterosexualidad y el significarse como masculino en su capacidad de reproducción y lo segundo se significa a través de la capacidad que, como hombre (en los términos del sistema sexo-género predominante), tiene para dar el sustento a su esposa e hijos. Es decir, estos elementos se transforman en mandatos.

Los mandatos o dimensiones de la masculinidad propuestos por Valdés y Olavarría (1997), ofrecen un punto de partida para este estudio, sin embargo, se comprende que éstas no son las únicas ni mucho menos las más importantes.

Derivado de las transformaciones socioeconómicas y culturales (como el ingreso de las mujeres al mercado de trabajo remunerado, educación superior, desempleo de los hombres y políticas de apoyo a las mujeres) los hombres vieron cuestionada su autoridad, fundamentada en su desempeño como proveedores únicos y su supuesta pertenencia al ámbito de lo público (Hernández, 2012; Minello, 2002). Estas condiciones dieron pie a estudios interesados en conocer y comprender el impacto de esos cambios en las identidades de los hombres (García \& Oliveira, 2005). Sin embargo, aunque se realizaron trabajos de gran calidad etnográfica, las investigaciones no alcanzaron a ahondar en los aspectos más amplios que involucran una perspectiva de género (Núñez, 2016; Hernández, 2012; Minello, 2002).

En diversos estudios realizados en México y Argentina se ha documentado una mayor propensión de las esposas e hijos a trabajar, como estrategia de supervivencia, cuando los jefes de familia han dejado de percibir ingresos o cuando estos disminuyen (García \& Pacheco, 2000; García \& Oliveira, 2007; Geldstein, 2004). Es importante explorar, no sólo los procesos económicos sino también el significado de la disminución o pérdida de ingresos en los varones 


\section{El trabajo como elemento constitutivo de la masculinidad}

que antes habían sido reconocidos como jefes económicos de la familia. A las transformaciones en su autopercepción como varón, se suman los cambios familiares ante esta nueva situación. Se plantea una posible redefinición de los papeles o funciones sociales reconocidas para varones y mujeres, así como posibles conflictos entre los valores normativos tradicionales y los emergentes (Burin et al., 2007).

Entre los mixtecos de SMG es posible identificar diferentes procesos en los que el contexto migratorio ha propiciado nuevas alternativas de participación de las mujeres en el ámbito público, por ejemplo el acceso al mercado laboral, y entre los hombres un mayor acercamiento con el ámbito privado o doméstico; lo que implica nuevas maneras de vincularse con otras perspectivas de construcción y significación de la masculinidad y de la feminidad. Empero, sin deshacerse de la distribución inequitativa de poder que determina situaciones dialógicas, misma que se hace visible a través de las expresiones de los individuos durante las interacciones.

En este sentido, se comprende que la masculinidad no es una construcción dada, sino que se transforma y que la interacción y práctica social la configura, es decir, es producto del género. Ante esto, reflexionamos sobre cuáles son los procesos a través de los cuales se construye la masculinidad entre este grupo en Tijuana; y sobre los significados que otorga a la misma en diferentes espacios, contextos y elementos de socialización y objetivación que orientan las acciones de la vida diaria, particularmente las relaciones de género con las cuales es posible identificar la construcción de identidades masculinas y sus posibles transformaciones culturales entre algunos mixtecos en una ciudad fronteriza como Tijuana.

\section{Cuestiones Metodológicas}

Se reconoce la existencia de una pluralidad de maneras de construir la masculinidad y diversas maneras de significarla. No obstante, como indican Gutmann (2000) y Salguero (2006), aun cuando nos enfrentemos a la diversidad, existen al mismo tiempo semejanzas entre hombres que comparten ciertas experiencias socioculturales e históricas, pero esas semejanzas y esos hombres deben ser claramente situados dentro de un contexto histórico, "de ahí que sea necesario investigar las prácticas cotidianas en las cuales se involucran los varones, los conflictos y contradicciones a las que se enfrentan en su actuación como hombres, en un flujo procesal, en una configuración particular y durante un periodo específico y no como algo permanente" (Salguero 2006, p. 49-50).

A través del método etnográfico se buscó conocer cómo los hombres mixtecos de SMG radicados en Tijuana construyen y significan sus masculinidades a partir de las acciones e interacciones vinculadas al trabajo, se utilizaron las técnicas de observación directa y participativa en los lugares de convivencia y, en algunos lugares de trabajo, entrevistas a algunos varones de la comunidad mixteca en cuestión. El trabajo de campo se realizó durante los meses de junio y diciembre de 2015 y los meses de octubre y noviembre de 2017, en las colonias Camino Verde, Sonoita, Zapata, Cañada, El Pípila, Nueva Esperanza, todas ubicadas en la zona este del municipio de Tijuana. Así mismo, en lugares públicos como el Parque Sor Optimista ubicado en la zona conocida como la 5 y 10.

Se utilizó la técnica de la observación directa haciendo hincapié en la interacción que los varones realizan con otros varones ya sean clientes o sus propios empleados, así mismo se observó la manera en que realizan sus trabajos a partir del uso de las herramientas y expresiones corporales durante la realización del mismo y durante las interacciones sociales. 
Se puso primordial atención a la manera en cómo los hombres mixtecos ejercen liderazgo y mando al momento de trabajar, así como la manera en que se desenvuelven. La mayor parte de ellos se dedica a labores relacionadas con la construcción, otros son mecánicos y otros trabajan en servicios (meseros, jardineros y seguridad), no se encontró a algún hombre que trabajara en la industria maquiladora (las mujeres mixtecas tienen una mayor participación en dicha industria junto con el servicio doméstico). La mayor parte de los hombres con los que se habló son sus propios jefes y tienen a su cargo hasta cuatro trabajadores oriundos de la misma comunidad.

La información consta de discursos, de los propios informantes en torno al trabajo y la manera en que los hombres consideran se debe trabajar y para qué se debe trabajar, obtenidos a través de las charlas, así como de sus prácticas observadas en los lugares de trabajo y de convivencia. Algunos relatos dan cuenta de cómo el conocimiento o saberes necesarios para realizar los diferentes trabajos son ensalzados para que los hombres puedan aducir a una cierta superioridad ante otros hombres. A partir de esto, se realizó análisis de los discursos y prácticas usando al género como categoría de análisis, se examinaron en cada uno de ellos las relaciones de poder visibles a través de los símbolos, las normas, las instituciones, la organización social y la identidad/subjetividad que implican la diferenciación de cada una de las actividades laborales realizadas por algunos oriundos de SMG radicados en Tijuana.

Cuadro 1. Perfil de los informantes

\begin{tabular}{llllr}
\hline & Ocupación & $\begin{array}{l}\text { Tiempo de residencia en } \\
\text { Tijuana (años) }\end{array}$ & $\begin{array}{l}\text { Experiencia migratoria } \\
\text { internacional }\end{array}$ & $\begin{array}{r}\text { Estado } \\
\text { civil }\end{array}$ \\
\hline César & Mecánico & 20 & Sí & Casado \\
\hline Raúl & Construcción & 17 & Sí & Casado \\
\hline Margarito & Contratista & 4 & Sí & Casado \\
\hline Sergio & Empleado & 16 & No & Casado \\
\hline Héctor & Albañilería & 17 & No & Separado \\
\hline Leonardo & Losetero & 13 & No & Caudo \\
\hline Rodrigo & Electricisita & 15 & No & Casado \\
\hline Raúl J & Jardinero & 3 & Sí & Casado \\
\hline Miguel & Contratista & 30 años viviendo en & Sí & \\
& & Estados Unidos & & Casado \\
\hline Pancho & Comerciante & 30 & Sí & Casado \\
\hline Cirilo & Albañilería & 25 & Sí & Separado \\
\hline Lucio & Albañilería & 15 & No & \\
\hline
\end{tabular}

Fuente: elaboración propia con base en información de trabajo de campo.

\section{Mixtecos en Tijuana: masculinidad y trabajo en el contexto fronterizo- migratorio}

En Baja California hay un número significativo de migrantes procedentes de Guerrero y Oaxaca que se adscriben a la etnia mixteca (Velasco, 1995). La llegada de migrantes mixtecos a principios de los años setenta coincidió con el auge agrícola de los campos de riego en estados como Sinaloa, Sonora y Baja California, en donde destaca el Valle de San Quintín (Cárdenas 2006, p. 17). En Baja California se asentaron comunidades procedentes de diferentes regiones del sur de México, "La población migrante en el estado, de origen indígena, es principalmente mixteca de Oaxaca, pero también Triqui y Zapoteca de Oaxaca, mixteca y náhuatl de Guerrero" (Velasco Ortiz et al., 2014, p. 81). 


\section{El trabajo como elemento constitutivo de la masculinidad}

El proceso migratorio vivido por los mixtecos es en sí un elemento de análisis en términos de construcción de masculinidades, pues desde la dimensión de la masculinidad que hace referencia al trabajo se avizora la necesidad de proveer a las familias, lo cual contribuye a dar un significado a la masculinidad entre varones mixtecos. Además, la idea de acudir a lugares distantes de su lugar de origen podría generar entre los mixtecos la idea de que migrar es un rito de paso para convertirse en verdaderos hombres.

Tijuana ha tenido procesos migratorios y de asentamiento particulares. Por un lado, por su ubicación geográfica se consolidó como una ciudad urbana e industrial que basa su producción en la intensidad de mano de obra, particularmente en las maquilas(Velasco, 1995 y 2016). Así mismo en la ciudad se viven cotidianamente procesos transfronterizos entre los que se visualizan cruces internacionales (Narváez, 2007), ya sea con fines laborales o escolares que de alguna manera pueden impactar en el tipo de intercambio cultural y simbólico durante las interacciones sociales entre los mixtecos.

La colonia Camino Verde, ubicada muy cerca de la zona conocida como 5 y 10, es considerada, en términos geográficos, la zona central de Tijuana, además de ser una zona comercial muy concurrida, es emblemática por ser donde se asentaron a mediados de la década de 1990 las primeras familias que llegaron de SMG a Tijuana. Sin embargo, es posible encontrarles en las colonias: La Cañada, El Pípila, Emiliano Zapata y Nueva Esperanza, todas ellas ubicadas al este de la ciudad. Los procesos de asentamiento son dignos de resaltar, pues fue a través de la organización articulada por la identidad étnica que diversos grupos de mixtecos obtuvieron un terreno en el que construyeron su casa (Ángeles, 2012, p. 45).

La migración mixteca, como muchas en otras en el país, es de índole económica. Es decir, la movilidad se da como respuesta a entornos paupérrimos en donde las oportunidades de crecimiento económico y desarrollo, en términos de bienestar, son limitadas. Sin embargo, el apego que se tiene a trabajar la tierra forma parte de un imaginario social pero también de una realidad. Por ejemplo, en los lugares de origen de los mixtecos el trabajo agrícola, sembrar la tierra y el barbecho construyen una imagen del hombre indígena campesino. Ahora bien, en el entendido de que se busca conocer el proceso de construcción de la masculinidad, así como comprender el significado de la misma entre los hombres mixtecos en Baja California, y considerando que el trabajo es una dimensión en las que se fundamenta la masculinidad, es necesario evidenciar: por un lado, las formas de empleo y trabajo que desempeñan los hombres mixtecos de SMG en Tijuana y confrontarlo con las actividades que se desarrollaban en el pueblo de procedencia; y por el otro, en términos económicos, la pobreza y los bajos salarios son factores que promueven estrategias de subsistencia, mediante las cuales, las familias involucran a más de uno de sus miembros en actividades económicas remuneradas. En este sentido, el trabajo remunerado tanto de hombres como de mujeres podría permitir ajustes en la organización familiar entre ellos la participación de los varones en el cuidado y crianza de su prole.

La llegada de migrantes mixtecos a principios de los años setenta coincide con el auge agrícola de los campos de riego en estados como Sinaloa, Sonora y Baja California, en donde destaca el Valle de San Quintín (Cárdenas 2006); pues éste cuenta con la característica de ser "la única región de Baja California donde la población indígena tiene un peso significativo. [...] Esta población indígena, de origen migrante, es principalmente mixteca de Oaxaca, pero también triqui y zapoteca de Oaxaca, mixteca y náhuatl de Guerrero" (Velasco, Zlolniski y Coubés, 2014, p. 81). 
En este sentido, las primeras grandes oleadas migratorias realizadas por estos grupos pueden ser situadas, en términos culturales en un entorno rural al que acudían los hombres a trabajar en las actividades agrícolas, las cuales son un referente masculino en las comunidades étnicas en México. En consecuencia, ameritaría ahondar en el tema de trabajo como dimensión de la masculinidad a través de la cual los varones la significan.

La cuestión del trabajo, la migración, la generación y la experiencia migratoria internacional conforman también traslapes con los cuales las expresiones y significaciones masculinas se hacen presentes. Por ejemplo, realizar cualquier tipo de actividad económica como puede ser jardinería, pintura, albañilería o mecánico, al menos derivado de las observaciones en campo, no son motivo de competencia masculina, es decir, todos los empleos son bien vistos. La diferencia surge en cuanto los sujetos han cruzado la frontera, pues algunos varones consideran que trabajar en Estados Unidos genera mejores ingresos y que sus familias pueden tener mejores condiciones de vida en Tijuana. De ahí que la idea de cruzar al vecino país sea constante y que genere tensiones familiares en torno a las decisiones de cruzar. Otro de los cambios significativos está en función de la división sexual del trabajo, pues con la participación de las mujeres en actividades económicas, aun en condiciones de precariedad e informalidad trastoca la ordenanza del sistema sexo-género de los mixtecos en la región.

La mayor parte de los varones se dedica a labores relacionadas con la construcción, otros son mecánicos y otros más trabajan en servicios (meseros, jardineros y seguridad). Durante el periodo del trabajo de campo no se contactó a algún hombre que trabajara en la industria maquiladora. Solamente a mujeres mixtecas quienes tienen una mayor participación en dicha industria, junto con el servicio doméstico. La mayor parte de los hombres con los que se ha hablado son sus propios jefes y por lo menos uno emplea a otros varones oriundos de la misma comunidad.

Entre algunos mixtecos de San Miguel El Grande, el trabajar va más allá de la mera obligación o mandato de ser el proveedor. A través del trabajo en el campo, la comunidad perpetúa la idea de que los hombres son los únicos capaces de realizar las actividades agrícolas; ya sea por la complexión física, que supone una mayor resistencia a los trabajos pesados que implica el trabajo agrícola o bien por las condiciones cognitivas que implica el mismo. Este sistema sexogénero implica que a través de su contraparte femenina los hombres validan su masculinidad sobreponiendo su actividad laboral por encima de las actividades realizadas por las mujeres. Entre los varones mixtecos en SMG, la división sexual del trabajo está naturalizada y son evidentes los roles en torno al trabajo de hombres y mujeres.

De acuerdo con la información proporcionada por Consta, cuando le pregunté sobre cómo era su vida en SMG, me relató que los varones adultos son los encargados de la siembra del campo. Además, la agricultura, es decir, el trabajo en el campo es básicamente la única actividad que realizan los hombres adultos padres de familia en la comunidad de origen. Esta situación es muestra de la división sexual del trabajo así como de la naturalización que se hace de los cuerpos de los hombres y de las mujeres; con la cual se asume que el trabajo del campo es difícil, pesado y que sólo los hombres, por su complexión física, lo pueden realizar. En términos de Rubin (1986, p. 97.) esto es una muestra de cómo las relaciones entre hombres y mujeres actúan en función de un sistema sexo-género "determinado por el conjunto de disposiciones por el que una sociedad transforma la sexualidad biológica en productos de la actividad humana". Además, a partir de este hecho, es posible visualizar cómo al trabajo en el campo, realizado por los varones, se le otorga una valía superior, pues todos los demás 


\section{El trabajo como elemento constitutivo de la masculinidad}

miembros de la familia asumen que este trabajo es importante y valioso, razón por la cual no se cuestiona que los varones no realicen otra actividad que no sea esa. Se considera que el conocimiento que este trabajo requiere es tal, que sólo los hombres son capaces de tener. Lo que por un lado lleva a los hombres a realizar este trabajo y a sentir gusto u orgullo por hacerlo.

Así mismo, a los niños y jóvenes varones se les asignan tareas fuera del hogar, los niños se encargan de juntar leña mientras que los jóvenes son los encargados de ayudar en las tareas del campo a sus padres. De esta manera se da un escalamiento en el trabajo que estos realizan en función de la edad; los mayores asumen la responsabilidad de trabajar la tierra cuando los padres consideran que están en edad de ser responsables. Esta situación me lleva a inferir que las labores domésticas son realizadas por las mujeres y niñas, lo que supone un mayor control sobre la movilidad de las mujeres en los espacios públicos. Por ejemplo, la cosecha de maíz y frijol rara vez es puesta en el mercado de manera directa.

Consta, una mujer de 37 años, oriunda de San Miguel, me contó que las familias no se alimentan únicamente de estos productos todo el año, que el maíz es transformado en tortillas por las mujeres, quienes previamente se encargaron de nixtamalizarlo, de preparar la masa y de hacer tortillas. Las tortillas se preparan en la casa y las mujeres salen al mercado un día a la semana (en el que llegan vecinos de otras localidades a ofrecer sus productos) a hacer trueque por lo que ellas consideran que les hace falta en su hogar. Pero las mujeres no sólo se encargan de eso, sino que también crían pollos para poder intercambiarlos durante los días de mercado. Además de los trabajos de cuidado y de alimentación para la familia. Aun cuando este trabajo proporciona sustento al hogar el trabajo en el campo, el trabajo de la mujer no es reconocido como el del hombre.

Estas actividades implican saberes y prácticas específicas que son del dominio de las mujeres, también a través de ellas se obtienen recursos tanto monetarios como en especie. Ante lo cual deben ser considerados como tal en el mismo nivel que el trabajo de la tierra realizado por los hombres. Sin embargo, no es considerado como un trabajo que sea motivo de expresiones simbólico-culturales como la composición de canciones, como lo es el trabajo de los hombres en el campo. Al menos durante el trabajo de campo no encontré evidencia de ello.Por lo tanto, el trabajo masculino en el campo adquiere un valor simbólico que se enaltece al ser catalogado como el principal sustento de la familia.

Sin embargo, el alejamiento del trabajo en el campo implica que algunos varones mixtecos están dispuestos a cambiar de actividad para cumplir como proveedores ante su familia y su comunidad y al mismo tiempo retomar elementos como el conocimiento, la habilidad y valentía para significarse ante otros hombres. Así que al migrar anteponen la proveeduría y bienestar de su propia familia realizando trabajos que no necesariamente cumplen con lo establecido culturalmente en su comunidad. Pero que en términos de hombría les significan ante otros hombres.

Las principales actividades que realizan los varones de este grupo de mixtecos fuera de su lugar de origen, particularmente en Tijuana, están relacionadas con la construcción. Algunos son albañiles, yeseros, loseteros. Es decir, la construcción tiene diferentes aristas y no se remite únicamente a la construcción de paredes con ladrillos. También hay otros que se dedican a la jardinería y podado de árboles, así como los que dan mantenimiento a inmuebles cuyas actividades van desde la plomería, pintura e impermeabilizar. 
Por otro lado, la participación de las mujeres en actividades remuneradas es amplia. La mayoría de ellas realiza trabajo doméstico en casas de sectores con mayor ingreso económico. Al preguntarle a estas mujeres a qué se dedican, ellas suelen responder: "trabajo en casa". Esta respuesta al principio del trabajo de campo me llevaba a malinterpretar su actividad pues yo pensaba que se dedicaban a las labores domésticas de su hogar. Sin embargo, la respuesta "trabajo en casa" hace referencia a que realiza un trabajo doméstico remunerado fuera de su hogar. Otra parte de las mujeres de origen mixteco trabaja en la industria maquiladora, algunas cumplen con horarios específicos de 7 u 8 horas y otras además tienen rotación de turnos de manera semanal.

Los espacios de trabajo tanto de hombres como de mujeres son diferenciados. Los hombres cumplen con sus trabajos organizando su tiempo en función de la distancia y del tipo de trabajo que requieren hacer; además, tienen mayor movilidad en el espacio público de la ciudad mientras que las mujeres están confinadas a espacios cerrados y con mayores exigencias en cuanto a horarios y supervisión. Sin embargo, el hecho de que las mujeres puedan ser controladas en torno a su movilidad y a sus relaciones interpersonales no impide que algunas de ellas, al contribuir económicamente para satisfacer las necesidades domésticas, exijan a los varones a realizar actividades que en sus lugares de origen no hacían, tales como la preparación de alimentos o el cuidado y atención de los hijos. Entre algunos grupos sociales, como esta comunidad mixteca, la experiencia migratoria, aunado a la inserción de las mujeres en el mercado laboral, ha propiciado que algunas mujeres exijan a los varones su participación en algunas labores domésticas, particularmente en lo concerniente al cuidado de los hijos.

Sergio e Iraís son una pareja que sirve como un ejemplo de lo anterior. Ellos tienen tres hijos, uno de seis, otra de ocho años y un bebé de un año. Sergio trabaja como ayudante general con Margarito; el ser ayudante general tiene la característica de no contar con una especialización en un oficio, algunos días está pintando, otro hace la mezcla de cemento. Iraís trabaja en una maquiladora, tiene que cumplir con un horario de 8 horas diarias y además rota turnos de manera semanal. Previo a que Iraís cumpliera su cuarentena posterior al parto, le pregunté sobre cómo haría con el cuidado del bebé, sobre todo durante las noches que ella trabajara y ella me respondió que esa iba a ser la responsabilidad de Sergio. "Yo creo que va a volver[se] loco" me comentó mientras se reía.

La respuesta de Iraís a mi pregunta me hace reflexionar ante los cambios en las relaciones de género suscitados en Tijuana con respecto al lugar de origen. Es decir que, mientras en SMG las mujeres realizaban todas las tareas domésticas, en Tijuana la participación de algunos hombres en dichas tareas se ha incrementado. Iraís asume que Sergio, su esposo, se tienen que hacer cargo del cuidado de los hijos mientras ella trabaja. En esta situación, en la que algunas mujeres fuera de su lugar de origen ingresan al mercado laboral da pauta para que algunos hombres participan más activamente en las labores domésticas, como es el caso de Sergio; quien se hace cargo de preparar desayunos para sus dos hijos en edad escolar, además de atender al bebé, esto me lo ha contado él mismo, además de que a través de sus publicaciones en Facebook, se toma fotos mientras cocina o limpia la casa. Sergio recurre a mostrarse como un hombre que colabora en la casa y a partir de esa imagen que trata de proyectar construye y significa su masculinidad. A decir de él, un hombre debe colaborar con las tareas del hogar y asume que en los lugares de origen los hombres actúan de manera machista. 


\section{El trabajo como elemento constitutivo de la masculinidad}

Con el caso de Sergio se puede mostrar la existencia de un modelo masculino esperado por algunos mixtecos de SMG en Tijuana. Éste se construye a partir de dos elementos, el primero es el trabajo que, como he tratado de mostrar, va más allá de trabajar para proveer y a través de este se muestran saberes y valentía. Y el segundo, es hacer ver a su familia, a su esposa e hijos, que su trabajo es el más valioso y por lo tanto es lo único que los hombres deben hacer. Por lo tanto, las actividades domésticas tienen que ser realizadas por las mujeres o los hijos.

Aunque no percibí que hubiera comentarios positivos o negativos (como por ejemplo llamarle mandilón) por parte de otros hombres referentes a la situación de Sergio, considero que al mostrarse en las redes sociales como un hombre que "colabora" con las tareas del hogar busca justificarse ante otros varones mostrándose como un hombre que no es machista por hacer actividades que en los lugares de origen los hombres no realizan y que otros hombres en Tijuana no hacen o no se atreven a hacer en público. Sin embargo, aunque puedo constatar con otro caso, el ejercicio de violencia de género, Sergio ha sido el único varón que vi golpear a su esposa, a Iraís, en público. Ese ejercicio de violencia es muestra de la necesidad que Sergio tiene de mostrarse ante otros hombres como el hombre que manda y que se hace respetar en su casa.

De esta manera, y ante la diversidad de actividades que realiza Sergio en su empleo, es decir, que no realiza un trabajo con el que pueda hacer notar autonomía, conocimiento o valentía infiero que entre algunos varones mixtecos de SMG tener un trabajo con el que tengan la posibilidad de mostrar a otros hombres y mujeres que son buenos, que nadie más lo puede hacer mejor, y que además se les retribuye bien económicamente es parte de elemental de la construcción de la masculinidad de estos mixtecos en torno al trabajo.

Considero que los hombres mixtecos no están buscando tener trabajos de estatus alto a nivel social sino más bien el estatus está dado entre los hombres de la misma comunidad y está en función del tipo de trabajo especializado que realizan, del ingreso y de quién tiene las habilidades y/o valentía para hacerlo.

Dos casos que me hicieron reflexionar al respecto fueron el de César, quien se mostró orgulloso al contarme que él es el único que puede arreglar carros Audi y BMW en Tijuana, y el de Raúl, un jardinero que me habló de la valentía necesaria para subirse a una palmera para podarla.

Con el pretexto de pedirle una asesoría sobre mi auto, le pedí a César que me diera la dirección de su taller y el aceptó con gusto. Un sábado poco antes del mediodía llegué a la colonia El Murúa, cerca de la Central de Autobuses de Tijuana, en busca del taller mecánico. Como punto de referencia me dijo que vería dos carros de marca Audi con placas americanas. No me fue complicado dar con el lugar y los autos me permitieron asegurar que estaba en el taller mecánico correcto.

César desmontaba el motor de otro auto al interior de su taller (que es el mismo lugar en donde se ubica su casa). Al preguntarle por los carros marca Audi, noté cómo erguía su cuerpo y con cierta altivez me dijo que se los traían unos "gabachos" porque en Estados Unidos repararlos sale muy caro y que él, al haber trabajado en un taller mecánico en aquel país, había adquirido el conocimiento especializado para poder repararlos. Me dijo que además de cobrar en dólares ese trabajo no lo hacía nadie más en Tijuana lo que le permitía cobrar lo que él quisiera. 
Continuando con las muestras que los varones me dieron respecto a su trabajo y la especialización de este, el caso de Raúl quien trabajó como jardinero en Estados Unidos también es evidencia de los discursos de especialización laboral. Me hice invitar por Raúl a acompañarlo un día para observar cómo hacía su trabajo y el día que acudí coincidió que era el día que tenía que podar un par de palmeras en la colonia Playas de Tijuana.

Raúl narró que había pasado por un proceso de deportación y que su esposa e hijas se habían quedado en California, él trabajaba como jardinero. Casi con la voz entrecortada me platicó lo difícil de ese proceso y me comentó la alegría cuando pudo juntar dinero para comprar sus primeras tijeras de jardinería en Tijuana. Raúl me comentó que su trabajo implica tener conocimiento, paciencia y habilidad para poder hacer su trabajo de jardinería, pero puso particular énfasis cuando se refirió a podar las palmeras:

Ese trabajo no lo hace cualquiera, primero porque necesitas tener los huevos para subirte a la palmera, también debes tener buena herramienta y saber usarla [...] Me gusta mucho arreglar jardines, cortar el pasto, arreglar las plantas, pero cuando me sale el jale de podar las palmeras me gusta más porque sé que ese trabajo no lo hace cualquiera, no cualquiera se sube a los árboles. Y sabes qué, con dos o tres palmeras que me salgan a la semana saco lo de trabajar todos los días en los jardines. (Raúl, charla octubre 2017).

Por principio de cuentas, estas dos actividades laborales de César y Raúl muestran nuevamente un alejamiento de la actividad rural agrícola en la que quizá sus abuelos o padres fundamentaron su proveeduría y significaron su masculinidad. Los relatos sitúan a su propio trabajo como especializado y particularmente Raúl un grado superior de valentía para poder podar las palmeras.

Otro elemento de análisis es haber logrado cruzar hacia Estados Unidos. César manifestó que el haber trabajado en un taller mecánico en Estados Unidos le brindó la oportunidad de desarrollar un trabajo en Tijuana que nadie más es capaz de hacer, al menos entre los mixtecos de SMG. Por su parte, Raúl en Estados Unidos realizaba el mismo trabajo que hace en Tijuana. Sin embargo, al hacer referencia a su arrojo le abre las puertas para especializarse en su trabajo.

La migración, entonces, impacta tanto en las actividades de los hombres como de las mujeres, por lo tanto, la cuestión de la proveeduría en tanto significación de la masculinidad, queda rebasada. De tal manera que, la masculinidad, entre este grupo de mixtecos, construida en referencia a la especialización en el trabajo, genera entornos de competencia en donde el ganador es quien es capaz de demostrar que su trabajo no lo hace otro, es decir, que tiene y domina el conocimiento para hacerlo; que su trabajo es peligroso y que no todos tienen la valentía para hacerlo y por último, que les genera un ingreso mejor con el que pueden seguir siendo los principales proveedores de su hogar.

Finalmente, otro ejemplo que puede ayudarnos a comprender la valía que se le otorga al trabajo especializado entre algunos varones mixtecos de SMG, puede ser visto desde mi propia experiencia de participación en las diferentes actividades de gestión que el Comité de Deportes y la mayordomía me asignaban. En diferentes ocasiones, Margarito, el "Mayordomo 2016" de nombre Rodrigo y el presidente del comité deportivo, Francisco, me llamaban por teléfono para pedirme redactar solicitudes, ante las autoridades civiles, de dinero y permisos para uso de los espacios públicos. Cuando recibía una llamada de ellos y de entrada me llamaban 


\section{El trabajo como elemento constitutivo de la masculinidad}

"Licenciado" yo ya sabía que tenía un encargo que cumplir con la comunidad. Quizá era por imagen que ellos tenían de mí y de las características de un "Licenciado", como una persona con estudios, que acudían a mí para realizar ese trabajo. Aunque esta actividad no se ajusta plenamente a la conceptualización utilizada en esta tesis por no ser remunerada, sirve para mostrar como mi "expertise" era reconocido entre ellos.

En cierta manera, los varones reproducen la división sexual del trabajo de su comunidad de origen. Es decir, trabajan en entornos públicos, mientras que las mujeres desempeñan las labores domésticas y su trabajo remunerado se encuentra en espacios donde existe una estricta vigilancia y control. la necesidad de proveer a una familia llevó a algunos hombres de SMG, de ciertas generaciones a tomar la decisión de migrar para trabajar como jornaleros. Pero con el cambio generacional surgieron nuevas motivaciones para migrar y con ello la diversificación en los tipos de trabajo. Este mismo punto ayuda a respaldar la idea de que no todas las migraciones son específicamente económicas, y que hay algunos varones, miembros de esta comunidad, que forman parte de generaciones específicas que migran sin tener como motivación el proveer a una familia, es decir, a su esposa e hijos.

Dentro de este orden de ideas, podemos afirmar que la generación, la migración y el tipo de trabajo se vinculan entre los mixtecos a partir de diferentes procesos históricos (cuadro 2). ${ }^{4}$ Además los diferentes estudios (Cornelius, et al, 2009; Velasco, 2002; Anguiano 1993) hicieron notar que las migraciones de los mixtecos suscitadas entre $1942 \mathrm{y}$, por lo menos, hasta 1990 eran realizadas mayoritariamente por varones casados. De esta manera, el análisis generacional entre los mixtecos está en función de las diferentes políticas migratorias de Estados Unidos y/o políticas de desarrollo agrícola e industrial del gobierno federal en México. Así mismo, las actividades económicas y los lugares de llegada.

Cuadro 2. Generación, migración y trabajo entre los mixtecos

\begin{tabular}{|c|c|c|c|}
\hline Generación & Proceso histórico & Lugar de llegada & Tipo de trabajo \\
\hline $1942-1964$ & $\begin{array}{l}\text { Programa bracero (vinculado a } \\
\text { la II Guerra Mundial) }\end{array}$ & $\begin{array}{l}\text { Estados Unidos } \\
\text { (diferentes estados } \\
\text { de la Unión } \\
\text { americana) }\end{array}$ & Jornaleros \\
\hline $1970-1990$ & $\begin{array}{l}\text { Modernización y tecnificación } \\
\text { agrícola en México (vinculado } \\
\text { a Política gubernamental) }\end{array}$ & $\begin{array}{l}\text { Sinaloa, Mexicali y } \\
\text { San Quintín. } \\
\text { California en } \\
\text { EEUU }\end{array}$ & Jornaleros \\
\hline $1990-2000$ & $\begin{array}{l}\text { Efecto llamado (IRCA e } \\
\text { IIRIRA; leyes promulgadas por } \\
\text { Estados Unidos de legalización } \\
\text { de migrantes indocumentados) }\end{array}$ & $\begin{array}{l}\text { California y } \\
\text { Tijuana }\end{array}$ & $\begin{array}{l}\text { Construcción, jardinería, } \\
\text { servicios, }\end{array}$ \\
\hline $2000-2017$ & $\begin{array}{l}\text { Migraciones aventureras } \\
\text { (abandono institucional del } \\
\text { campo; incremento del nivel } \\
\text { educativo de algunos } \\
\text { miembros de la comunidad } \\
\text { mixteca) }\end{array}$ & $\begin{array}{l}\text { Diferentes áreas } \\
\text { urbanas en el país. } \\
\text { Tijuana }\end{array}$ & $\begin{array}{l}\text { Construcción, jardinería, } \\
\text { servicios. }\end{array}$ \\
\hline $\begin{array}{l}\text { Mixtecos nacidos en } \\
\text { Tijuana entre } 1990-2000\end{array}$ & Mayor nivel educativo & $\begin{array}{l}\text { Nacieron y viven } \\
\text { en Tijuana }\end{array}$ & $\begin{array}{l}\text { Profesionistas, } \\
\text { Profesores, }\end{array}$ \\
\hline
\end{tabular}

Fuente: elaboración propia.

${ }^{4}$ Este cuadro nos ayuda a identificar procesos estructurales que se vinculan estrechamente con las decisiones de migrar y con la diversificación de trabajos que los hombres estaban dispuestos a hacer. 
Sin embargo, a partir de los procesos migratorios y del cambio en las motivaciones para migrar podemos determinar dos factores: los individuales y los familiares, a través de los cuales los varones mixtecos deciden migrar o no migrar y/o dedicarse al trabajo agrícola o a cualquier otro. El factor individual puede estar condicionado por el estado civil o por las aspiraciones y expectativas individuales. Es decir, los varones de la generación que migró en los años posteriores al año 2000, eran solteros y realizan actualmente trabajos que no están vinculados al campo; mientras que los casados, en las generaciones anteriores, salían específicamente al trabajo como jornaleros (que de alguna manera los vincula al trabajo en el campo).

\section{Conclusiones}

El trabajo, como un elemento de la construcción y significación de la masculinidad, se ha transformado y con ello la significación de ser un hombre; en este documento fue posible evidenciar cómo existe una significación de la masculinidad respecto al trabajo diferente o más allá al aspecto de la proveeduría, es decir, existen perspectivas en el estudio de la masculinidad en donde se plantea que el vínculo entre trabajo y ser hombres responsables respecto a su familia es indivisible (Olavarría, 1997; Salguero, 2007; Viveros, 1997; Hernández, 2010).

Sin embargo, en este artículo se retomó la perspectiva en la que los hombres, a partir del trabajo, retoman los saberes, prácticas y valentía características que en este documento se ha denominado como "el desempeño laboral". Núñez (2007, p. 155) retomó este aspecto como "el saber hacer el trabajo" asiéndose de características como la malicia, el criterio, experiencia, destreza e inteligencia, a partir de las cuales los hombres hacen una valoración de su masculinidad. De esta manera, los hombres a partir de la competencia con otros hombres significan su masculinidad valorándose, como hombres, en función de las características de sus trabajos, la destreza y la valentía necesaria para hacerlos.

La cuestión del desempeño laboral es una consecuencia, considerando la inserción de las mujeres en el mercado laboral, que ha marcado la pauta para que la significación masculina como único proveedor se flexibilice y con ello los varones busquen nuevas maneras de significar el trabajo. Más allá de la perspectiva de Ángeles y Rojas (2000) quienes argumentan que la migración femenina es sólo una causa de la migración masculina, en este trabajo es posible observar cómo las mujeres migran sin tener una motivación de dependencia hacia los hombres. El caso de Consta y de Iraís constituyen vetas de análisis para indagar las motivaciones para migrar. Dando como resultado procesos de construcción de masculinidades diferentes a como los hacían en los lugares de origen.

En este tenor, se inserta la perspectiva generacional en torno al trabajo. Aunque, los varones de generaciones anteriores significaban su trabajo a partir de los medios que obtenían de este para proveer a sus familias. Sin embargo, es notoria la referencia que se hace en torno a la necesidad de contar con el conocimiento y la fuerza para hacerlo. Estos elementos han sido retomados por algunos varones de SMG radicados en Tijuana para construir su masculinidad a partir de los trabajos que realizan.

El apego al trabajo en el campo queda, entonces, como evocación a un pasado en los lugares de origen pues como vimos, hay una diversificación de las actividades laborales que los hombres realizan en Tijuana; mismas que han sido, en cierta medida, determinadas por la migración y momentos históricos específicos. 
En este aspecto, se hace notar cómo los varones mixtecos de SMG abrieron su panorama laboral en función de las opciones que el mercado ofrecía. Además, resignificaron el trabajo a partir de elementos como la especialización, la valentía y el conocimiento para competir con otros varones tanto de la misma comunidad como con otros de otros orígenes étnicos y mestizos.

También se evidencia el papel de las mujeres en la resignificación del trabajo en el ámbito de la proveeduría. Y además abre la posibilidad de explorar la participación de los hombres en las labores domésticas y de cuidado. Entre este grupo de mixtecos en Tijuana la división sexual del trabajo obedece a sistemas tradicionales en los que los roles de género están marcados por objetivaciones sociales en las que las mujeres están obligadas a realizar sólo ciertas actividades vedándoles el camino para tener una mayor movilidad y ser sometidas a control y supervisión en los lugares de trabajo por parte de los hombres y la comunidad.

Se alerta también que el ejercicio de la violencia por los hombres puede ser un acto que significa la masculinidad de algunos varones ante la incapacidad de insertarse en ámbitos de homosocialidad en donde la competencia está marcada por los elementos analizados en este documento en torno al trabajo. Otro aspecto que es necesario indagar es el papel de la educación en la resignificación de la masculinidad, en las motivaciones para migrar y en los cambios generacionales, tanto en los lugares de origen como en los de llegada. Pues en este artículo únicamente se deja entrever que puede ser un factor importante para la toma de decisiones entre hombres y mujeres de la comunidad mixteca de San Miguel El Grande.

\section{Referencias bibliográficas}

Ángeles, H., \& Rojas, M. (2000). Migración femenina internacional en la frontera sur de México. Papeles de Población, 23, 127-151.

Angeles, C. (2012). La educación como una estrategia para la movilidad social de la juventud mixteca en Tijuana. (TESIS) Maestría en Desarrollo Regional; El Colegio de la Frontera Norte, 150.

Anguiano, M. (1993). La migración de indígenas mixtecos / movilidad poblacional y preservación de identidades. DEMOS , 16-17

Burin, M., Jiménez, M., \& Meler, I. (2007). Precariedad laboral y crisis de la masculinidad. Impacto sobre las relaciones de género. In Precariedad laboral y crisis de la masculinidad. Impacto sobre las relaciones de género. Buenos Aires: Universidad de Ciencias Empresariales y Sociales.

Butler, J. (2001). El género en disputa. El feminismo y la subversión de la identidad. (Paidos, Ed.).

Cornelius, W., FitzGerald, D., Hernández-Díaz, J., \& Borger, S. (2009). Migration from the Mexican Mixteca: A Transnational Community in Oaxaca and California. La Jolla: Center for U.S.-Mexican Studies, University of California, San Diego.

Faur, E. (2004). Masculinidades y desarrollo social. Las relaciones de género desde la perspectiva de los hombres (UNICEF, Ed.). Arango Editores.

García, B., \& Oliveira, O. (2005). Fatherhood in Urban Mexico. Journal of Comparative Family Studies, 36(2), $305-327$.

García, B., \& Oliveira, O. (2007). Trabajo extradoméstico y relaciones de género: una nueva mirada. In M. Gutierrez (Ed.), Género, familias y trabajo: rupturas y continuidades. Desafios para la investigación politica. CLACSO, Consejo Latinoamericano de Ciencias Sociales.

García, B., \& Pacheco, E. (2000). Esposas, hijos e hijas en el mercado de trabajo de la Ciudad de México en 1995. Estudios Demográficos y Urbanos, 15(1), 35-63.

Geldstein, R. (2004). De "buenas" madres y "malos" proveedores. Género y trabajo en la reestructuración económica. Subjetividad y Procesos Cognitivos, 5, 126-155.

Gilmore, D. (1997). Cuenca mediterranéa: La excelencia en la actuación. In T. Valdés \& J. Olavarría (Eds.), Masculinidad/es Poder y crisis (pp. 82-90). FLACSO ISIS Internacional. 
Gutmann, M. (2000). Ser hombre de verdad en la Ciudad de México: ni macho ni mandilón. El Colegio de México.

Hernández, O. (2009). Descobijando a los hombres. Masculinidades y relaciones de género en Cd. Victoria. Universidad Autónoma de Tamaulipas.

Hernández, O. (2010). Neoliberalismo, masculinidades y exobreros en una ciudad mexicana. Revista de Antropología Experimental, 10, 245-255.

Hernández, Ó. (2012). Masculinidades en Tamaulipas una historia antropológica. Plaza y Valdés.

Minello, N. (2002). Los estudios de masculinidad. Estudios Sociológicos, XX(3), 715-732.

Narváez, J. (2007). Tijuaneados: jóvenes, informalidad y migración transfronteriza. El Cotidiano Revista de La Realidad Mexicana, 22(143), 57-62.

Núñez, G. (2016). Los estudios de género de los hombres y las masculinidades: ¿qué son y qué estudian? Culturales, IV(1), 9-31.

Olavarría, J. (1999). Adolescentes/jóvenes: qué poco sabemos de ellos. FLACSO.

Rubin, G. (1986). El tráfico de mujeres: notas sobre la "economía política" del sexo. Nueva Antropología, VIII (30), 95-145.

Salguero, A. (2006). Feminismo: masculinidad y paternidad. Tramas, 41-60.

Salguero, A. (2007). El significado del trabajo en las identidades masculinas. In O. Tena \& L. Jiménez (Eds.), Reflexiones sobre masculinidades y empleo (pp. 429-438). CRIM/UNAM.

Scott, J. W. (1996). El género: una categoría útil para el análisis histórico. El Género: La Construcción Cultural de La Diferencia Sexual, 265-302. https://doi.org/10.2307/1864376

Valdes, T. \& Olavarria, J. (1997). Masculinidad/es: Poder y crisis (pp. 1-88).

Velasco, L. (1995). Introducción. In S. González, O. Ruiz, L. Velasco, \& O. Woo (Eds.), Mujeres, migración y maquila en la frontera norte. El colegio de México - El Colegio de la Frontera Norte.

Velasco, L. (2002). El regreso de la comunidad: migración indigena y agentes étnicos. Los mixtecos en la frontera México-Estados Unidos. El Colegio de México - El Colegio de la Frontera Norte.

Velasco Ortiz, L. (2016). Reporte de resultados del estudio cualitativo en colonias: Proceso de asentamiento, integración social y reconstitución cultural entre poblaciones indígenas residentes en Ciudad Constitución, Municipio de Comondú, Baja California Sur.

Velasco Ortiz, L., Zlolniski, C., \& Coubès, M. L. (2014). De jornaleros a colonos. Residencia, trabajo e identidad en el V alle de San Quintin. El Colegio de la Frontera Norte.

West, C., \& Zimmerman, D. (1987). Doing Gender. Gender and Society, 1(2), 125-151. 